\title{
Oclusão Percutânea de uma Comunicação Interatrial Tipo Ostium Primum: Nova Opção Terapêutica ou Apenas um Golpe de Sorte?
}

\author{
Francisco Chamié', Daniel Silva Chamié de Queiroz², Jorge Luiz Haddad',
} Maria Luisa Meurer ${ }^{1}$, Maria Luisa Aragão ${ }^{1}$, Elephterios Sideris ${ }^{4}$

\section{RESUMO}

Até o momento, o uso percutâneo de próteses para o fechamento da comunicação interatrial tipo ostium primum (CIA OP) era considerado impossível pelo envolvimento e proximidade das valvas atrioventriculares. Os autores relatam, pela primeira vez, um caso bem-sucedido de oclusão percutânea de CIA OP utilizando Transcatheter Patch ${ }^{\circledR}$ (Custom Medical Devices - Atenas, Grécia). TST, sexo masculino, 25 anos, $80 \mathrm{~kg}$, portador de CIA OP e cleft mitral, sem regurgitação significativa. Operado em 2005, apresentou CIA OP residual de $16 \mathrm{~mm}$, recusando nova cirurgia. Em dezembro de 2007, foi encaminhado para tentativa de oclusão percutânea. O procedimento foi realizado sob anestesia geral e monitoração contínua por ecocardiograma transesofágico (ETE). O diâmetro estirado da CIA mediu $21 \mathrm{~mm}$, obtido pela oclusão do defeito com balão medidor CMD Sizing Balloon inflado com $6 \mathrm{ml}$ de solução salina e contraste. Sobre guia rígida, introduzida na aorta ascendente através do ventrículo esquerdo, foi posicionada bainha de Mullins $12 \mathrm{~F}$ através do defeito. Foi selecionado patch de tamanho médio, com cola cirúrgica de polietilenoglicol aplicada, montado sobre balão medidor CMD e introduzido através da bainha longa. O sistema foi recuado, ocluindo o defeito e expandindo o patch. Mantendo o sistema imobilizado, o paciente foi encaminhado para unidade de tratamento intensivo. O balão foi retirado sob fluoroscopia e ETE na manhã seguinte, deixando o patch aderido ao septo atrial. ETE de controle mostrou CIA OP completamente fechada. Mais estudos se fazem necessários para melhor estabelecer a segurança, a eficácia e a reprodutibilidade dessa técnica.

DESCRITORES: Defeitos do septo interatrial. Cateterismo cardíaco. Próteses e implantes.

1 Hospital dos Servidores do Estado, Ministério da Saúde - Rio de Janeiro, RJ.

${ }^{2}$ Instituto Dante Pazzanese de Cardiologia - São Paulo, SP.

3 Santa Casa de Ribeirão Preto - Ribeirão Preto, SP.

${ }^{4}$ Athenian Institute of Pediatric Cardiology - Atenas, Grécia.

Correspondência: Francisco José Araújo Chamié Queiroz. Av. Borges

de Medeiros, 3.501 - apto. 103 - Lagoa - Rio de Janeiro, RJ.

CEP 22470-001 - E-mail: fchamie@pobox.com

Recebido em: 13/9/2008 • Aceito em: 29/10/2008

\section{SUMMARY}

Transcatheter Closure of an Ostium Primum Septal Defect: Alternative Therapy or Just a Lucky Shot?

Up to now, transcatheter device closure of ostium primum atrial septal defects (ASD) was considered impossible due to the proximity of the AV valves. The authors report for the first time a successful case of catheter closure of an ostium primum septal defect using the Transcatheter Patch ${ }^{\circledR}$ (Custom Medical Devices - Athens, Greece). TST, male, 25 years old, $80 \mathrm{~kg}$, with ASD with mitral cleft, with no significant regurgitation. After surgery in 2005, presented residual ASD of $16 \mathrm{~mm}$. Refused new surgery. In December of 2007 was referred for attempted catheter closure. The procedure was done under general anesthesia and continuous monitoring using transesophageal echocardiogram (TEE). The stretched diameter of the ASD measured $21 \mathrm{~mm}$, obtained via occlusion of the defect with a CMD Sizing Balloon inflated with a $6 \mathrm{~mL}$ saline solution and contrast. Using a rigid guidewire introduced into the ascending aorta through the left ventricle, a Mullins $12 \mathrm{~F}$ sheath was positioned on the defect. An average-size patch was selected, polyethylene glycol surgical glue applied. It was mounted on a CMD Sizing Balloon and introduced through the long sheath. Then, the system was withdrawn, occluding the defect and expanding the patch. Keeping the system immobilized, the patient was referred to the intensive care unit. The balloon was withdrawn under fluoroscopy and TEE the following morning, leaving the patch adhering to the atrial septum. Control TEE showed that the ASD was completely closed. Further studies are needed to better establish the safety, efficacy and reproducibility of this technique.

DESCRIPTORS: Heart septal defects, atrial. Heart catheterization. Prostheses and implants.

O fechamento percutâneo da comunicação interatrial tipo fossa oval ou ostium secundum (CIA FO) tornou-se, universalmente, uma alternativa aceitável para a correção cirúrgica desse defeito. Acreditava-se, até o momento, que a oclusão percutânea da $\mathrm{ClA}$ tipo ostium primum (CIA $\mathrm{OP}$ ) não era viável em decorrência de sua proximidade e do envolvimento com as valvas atrioventriculares. Neste traba- 
Chamié F, et al. Oclusão Percutânea de uma Comunicação Interatrial Tipo Ostium Primum: Nova Opção Terapêutica ou Apenas um Golpe de Sorte? Rev Bras Cardiol Invas. 2008;16(4):489-494.

Iho, relatamos um caso de fechamento de uma CIA OP, utilizando um novo dispositivo percutâneo, o Transcatheter Patch ${ }^{\circledast}$ (Custom Medical Devices - Atenas, Grécia).

\section{RELATO DE CASO}

Paciente TST, sexo masculino, com 25 anos de idade e pesando $80 \mathrm{~kg}$, sabia ser portador de forma parcial de defeito do septo atrioventricular (DSAV) desde os cinco anos de idade. Era completamente assintomático sob o ponto de vista cardiológico. Seu ecocardiograma transtorácico (ETT) mostrava ampla CIA OP e valva mitral apresentando cleft e pequena regurgitação (Figura 1).

Permaneceu assintomático até março de 2005, quando teve seu defeito corrigido cirurgicamente.

Após a cirurgia, foi notada uma CIA OP residual de $16 \mathrm{~mm}$ (Figura 2), para a qual foi indicada nova cirurgia, terminantemente recusada pelo paciente.
Em dezembro de 2007, foi oferecida a opção de tentar fechar o defeito residual por meio de técnica percutânea com um novo dispositivo, o Transcatheter Patch $^{\circledR}$.

O paciente foi encaminhado ao laboratório de hemodinâmica para a realização do procedimento, que foi executado sob anestesia geral e monitorado por ecocardiograma transesofágico (ETE).

Após a confirmação do diagnóstico pelo ETE, foi obtido acesso venoso por punção da veia femoral direita e introdução de uma bainha de calibre 7 French (F). A seguir, foi administrada heparina na dose de 5.000 UI, seguida por doses adicionais de 2.500 UI a cada 30 minutos após a primeira hora de procedimento. Foram administrados, também, $2 \mathrm{~g}$ de cefalotina em bolo, seguidos por mais duas doses de $500 \mathrm{mg}$ no período pós-operatório imediato, para profilaxia antibiótica.

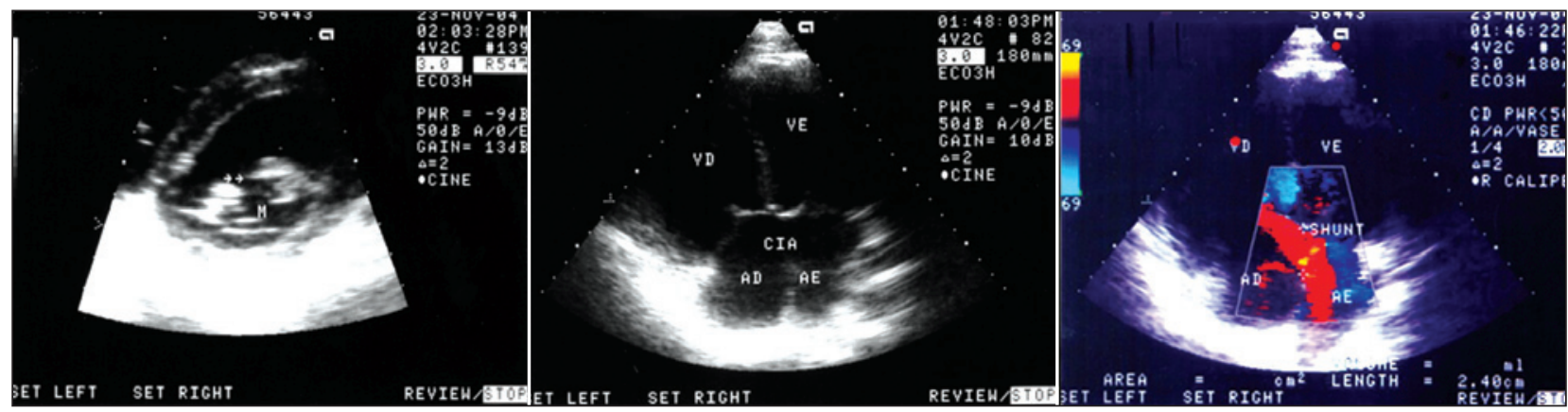

Figura 1 - Ecocardiograma transtorácico pré-operatório, no qual se observa, no painel à esquerda, o cleft da valva atrioventricular esquerda, típico dos defeitos do septo atrioventricular, apontando para a via de entrada. No painel central, o corte apical de quatro câmaras com os dois anéis atrioventriculares claramente individualizados e a comunicação interatrial tipo ostium primum. No painel à direita, observa-se fluxo em cores da esquerda para a direita através do defeito do septo atrial. $\mathrm{AD}=$ átrio direito; $\mathrm{AE}=$ átrio esquerdo; $\mathrm{CI} \mathrm{A}=$ comunicação interatrial; $M=$ mitral; $\mathrm{VD}=$ ventrículo direito; $\mathrm{VE}=$ ventrículo esquerdo.
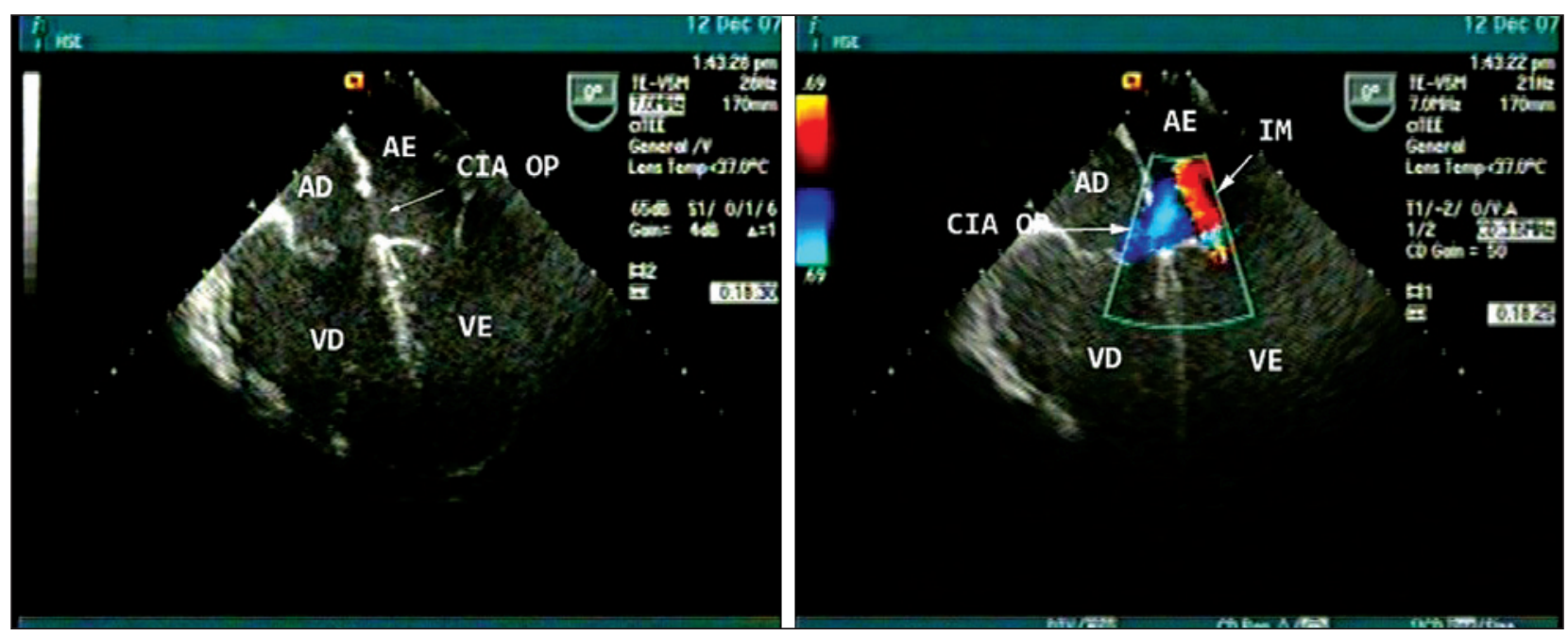

Figura 2 - Ecocardiograma transesofágico imediatamente anterior ao procedimento de oclusão, no qual se observa, no painel esquerdo, a comunicação interatrial residual pós-cirúrgica. À direita, observa-se fluxo em cores através da comunicação e pequena regurgitação pela valva atrioventricular esquerda. $\mathrm{AD}=$ átrio direito; $\mathrm{AE}=$ átrio esquerdo; $\mathrm{CI} \mathrm{A} \mathrm{OP}=$ comunicação interatrial tipo ostium primum; $\mathrm{IM}=$ insuficiência mitral; $\mathrm{VD}=$ ventrículo direito; $\mathrm{VE}=$ ventrículo esquerdo. 
Chamié F, et al. Oclusão Percutânea de uma Comunicação Interatrial Tipo Ostium Primum: Nova Opção Terapêutica ou Apenas um Golpe de Sorte? Rev Bras Cardiol Invas. 2008;16(4):489-494.

Um cateter diagnóstico multipropósito (MPA2) $6 \mathrm{~F}$ foi utilizado para o cateterismo direito e esquerdo, que revelou pressões normais na artéria pulmonar.

Apesar de presentes, foi impossível cateterizar qualquer veia pulmonar esquerda pela sua localização. Após várias tentativas infrutíferas, posicionouse uma guia longa, super-rígida (super-stiff), de 0,035" na veia pulmonar superior direita. Sobre ela, introduzimos um balão de oclusão CMD (Custom Medical Devices - Atenas, Grécia), que obteve a completa obliteração do defeito com $6 \mathrm{ml}$ de uma solução diluída de contraste, correspondendo a um diâmetro estirado de $21 \mathrm{~mm}$ (Figura 3).

Apesar de ter obtido o diâmetro estirado corretamente, a guia apresentou alguma instabilidade durante o processo e considerou-se que seria complicado executar o implante do dispositivo através de um trajeto com várias curvas fechadas sobre uma guia com pouca estabilidade. Optou-se, então, por posicionar a guia na aorta descendente, passando através do ventrículo esquerdo, o que foi conseguido com relativa facilidade.

Nessa nova posição, foi introduzida bainha de Mullins $12 \mathrm{~F}$ em átrio esquerdo, avançada com segurança sobre a guia através do defeito.

O dispositivo foi montado sobre balão de oclusão igual ao utilizado anteriormente. Antes da introdução, a "cauda" do patch foi embebida em cola biológica, à base de polietilenoglicol, e o dispositivo foi introduzido pelo interior da bainha longa.

Enchendo o balão com a mesma quantidade de solução de contraste utilizada para a oclusão, o balão foi inflado, expandindo o patch e provocando sua aderência às bordas do defeito (Figura 4).

Após confirmar a adequada posição do dispositivo e a ausência de shunt residual ao ETE, o corpo do cateter foi fixado à pele, na região inguinal, por meio de sutura com mononáilon 3-0, e o membro inferior

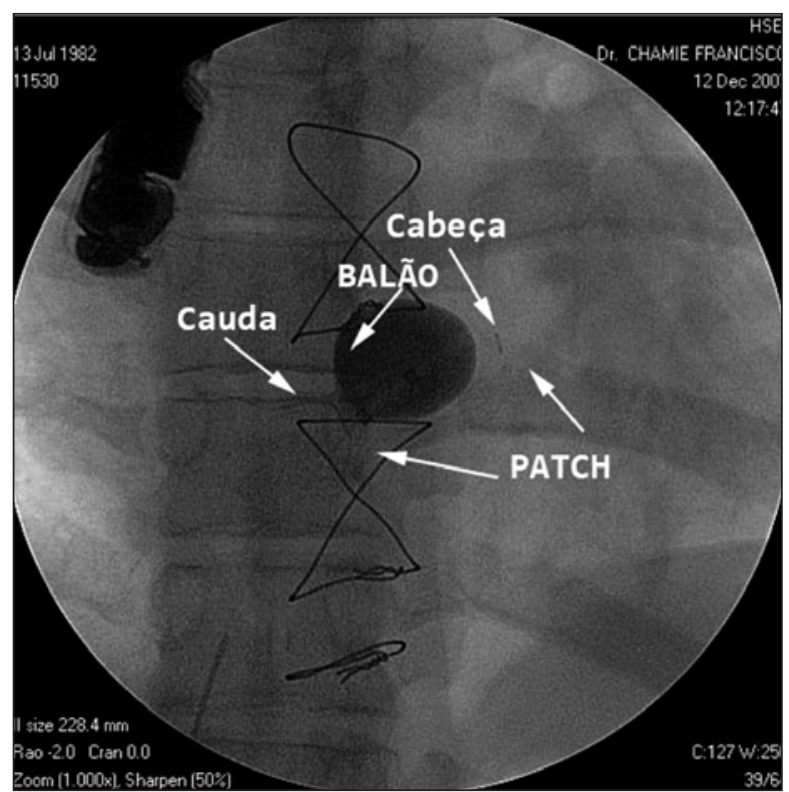

Figura 4 - Imagem fluoroscópica do balão inflado, demonstrando os limites do patch. Na seta da direita, o anel metálico na extremidade distal (cabeça); na seta da esquerda, um fino fio delimitando a extremidade proximal (cauda) do dispositivo; e no meio está o balão inflado. Os outros dois anéis radiopacos correspondem ao corpo do balão de oclusão.
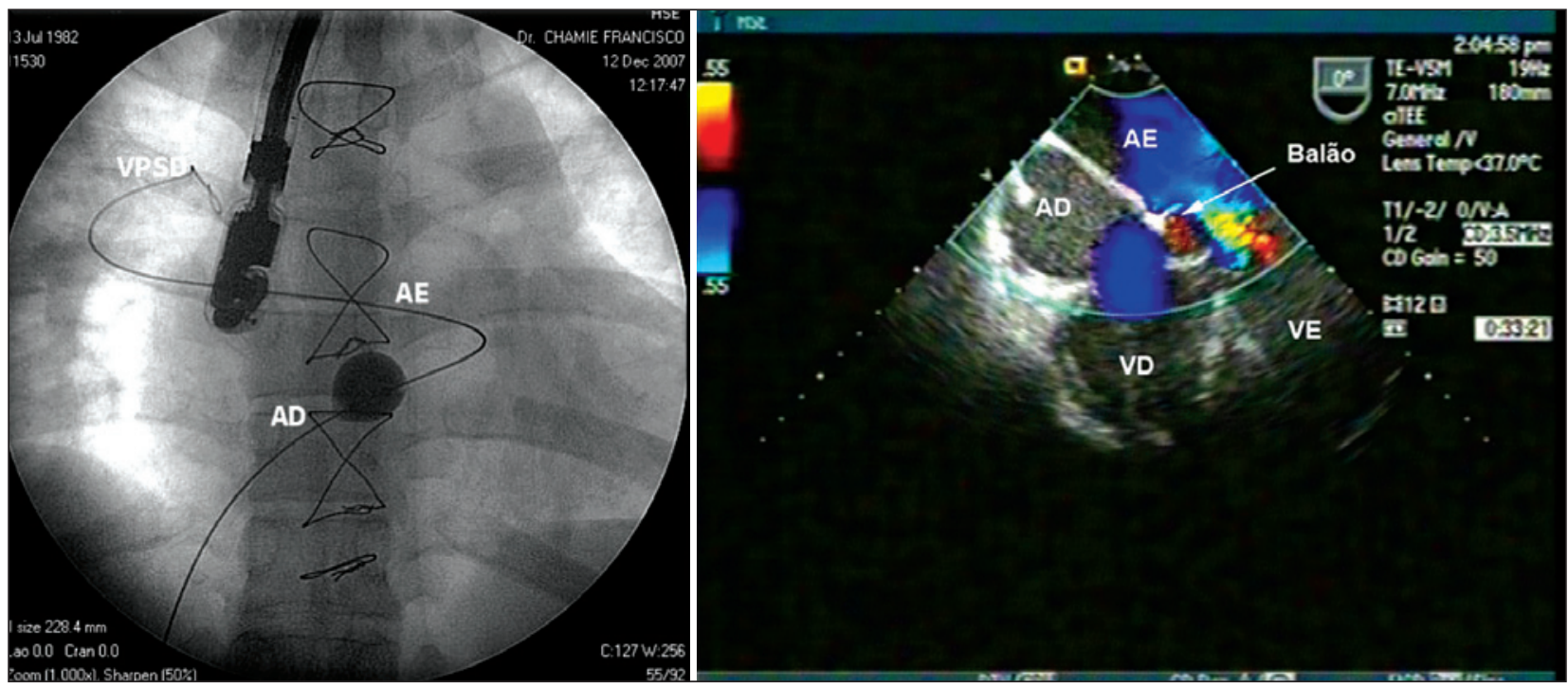

Figura 3 - À esquerda, observa-se imagem fluoroscópica da guia super-rígida colocada em veia pulmonar superior direita e o balão ocluindo o defeito. Observa-se a pequena deformação do balão entrando no orifício da comunicação. À direita, observa-se o ecocardiograma transesofágico mostrando o balão de oclusão no interior da comunicação interatrial tipo ostium primum e a ausência total de fluxo através do defeito pelo Doppler em cores. $\mathrm{AD}=$ átrio direito; $\mathrm{AE}=$ átrio esquerdo; $\mathrm{VD}=$ ventrículo direito; $\mathrm{VE}=$ ventrículo esquerdo; $\mathrm{VPSD}=$ veia pulmonar superior direita. 
Chamié F, et al. Oclusão Percutânea de uma Comunicação Interatrial Tipo Ostium Primum: Nova Opção Terapêutica ou Apenas um Golpe de Sorte? Rev Bras Cardiol Invas. 2008;16(4):489-494.

direito foi imobilizado, por um período aproximado de dezesseis horas, com o paciente internado em unidade de terapia intensiva. No dia seguinte, o balão foi desinflado e, cuidadosamente, removido sob monitorização angiográfica e por ETE no laboratório de hemodinâmica (Figura 5). Após a retirada do balão, o dispositivo se manteve em posição sem apresentar shunt residual ou interferência com as valvas atrioventriculares.

O paciente recebeu alta hospitalar no dia seguinte, sem intercorrências.

Os ecocardiogramas de seguimento demonstraram que o defeito estava completamente ocluído e a regurgitação mitral preexistente se manteve inalterada (Figura 6).

\section{DISCUSSÃO}

A CIA OP é, tradicionalmente, estudada junto com o chamado DSAV, porque a anormalidade da junção atrioventricular, com conseqüente alteração na arquitetura das valvas atrioventriculares, é a característica mais proeminente desses defeitos.

São classificados, na dependência da presença de um ou dois anéis atrioventriculares individualizados, em forma total ou forma parcial. Em ambas as formas, a CIA tem como assoalho os folhetos das valvas atrioventriculares.

Na forma parcial com dois anéis atrioventriculares individualizados, quase sempre a valva atrioventricular esquerda apresenta alterações anatômicas e funcionais (geralmente regurgitação), que, além da posição espacial da CIA OP desfavorável para abordagem transcateter, tornam a correção cirúrgica imperativa ${ }^{1}$.

As próteses atualmente disponíveis apresentam dois discos com esqueleto metálico (próteses de duplo disco, como Cardioseal/Starflex ${ }^{\circledR}, \mathrm{Helex}^{\circledR}$, $\mathrm{Cardia}^{\circledR}$, etc.) ou um elemento oclusor central e dois discos para fixação inteiramente feitos de liga metálica (próteses de rolha,
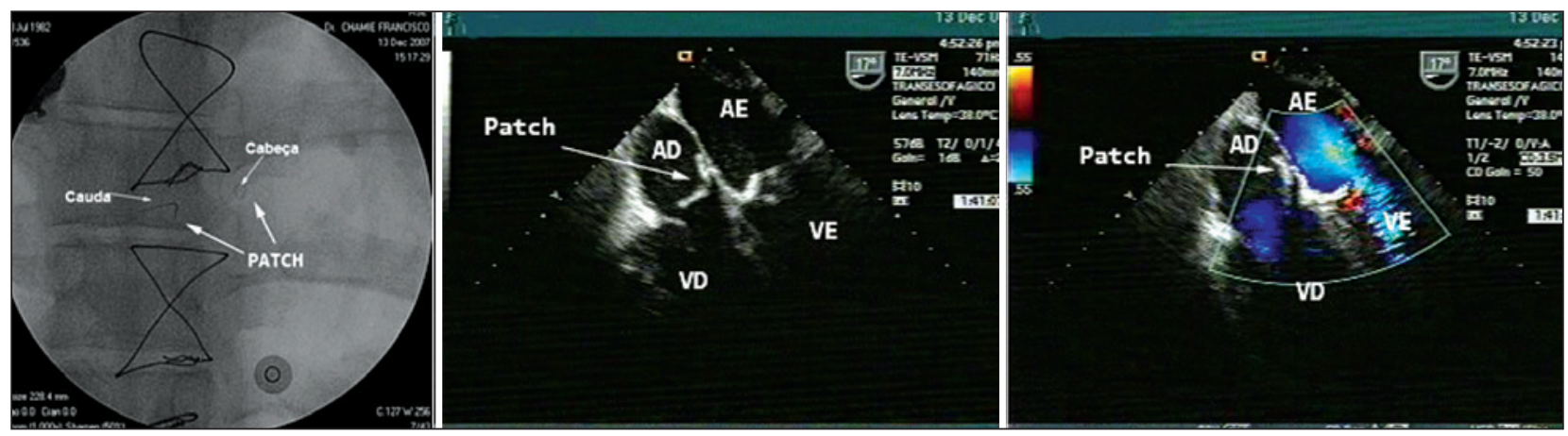

Figura 5 - À esquerda, imagem fluoroscópica do patch. Observa-se o pequeno anel demarcando a extremidade distal (cabeça) e os fios metálicos delimitando a extremidade proximal (cauda) do patch. Observa-se, nessa figura, que foram retirados o fio-guia e o corpo do cateter. No centro e à direita, imagens ecocardiográficas demonstrando o patch ocluindo o defeito e ausência de shunt residual pelo Doppler em cores. AD = átrio direito; $\mathrm{AE}=$ átrio esquerdo; $\mathrm{VD}=$ ventrículo direito; $\mathrm{VE}=$ ventrículo esquerdo.
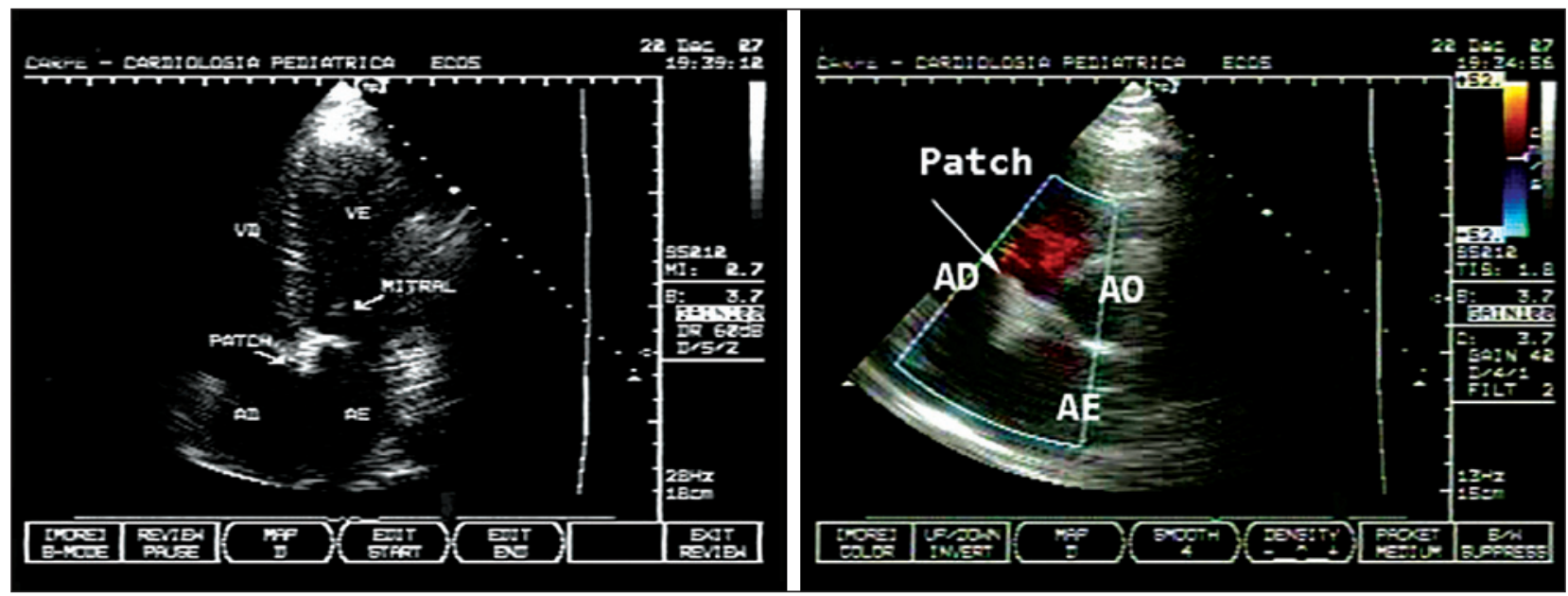

Figura 6 - Ecocardiograma transtorácico de seguimento. À esquerda, observa-se o patch em posição ocluindo o defeito. À direita, o Doppler em cores não capta fluxo residual pela comunicação. $A D=$ átrio direito; $A E=$ átrio esquerdo; $A O=$ aorta; $V D=$ ventrículo direito; $V E=$ ventrículo esquerdo. 

ou Apenas um Golpe de Sorte? Rev Bras Cardiol Invas. 2008;16(4):489-494.

como Amplatzer ${ }^{\circledR}$, Occlutech $^{\circledR}$, etc.). Em ambos os casos, a ausência total da borda inferior do defeito impede a fixação das próteses tradicionais, tornando impossível a abordagem terapêutica com cateter ${ }^{2,3}$.

O Transcatheter Patch ${ }^{\circledR}$ é um dispositivo em forma de luva, composto de espuma de poliuretano, adaptado sobre um balão de oclusão (duplo ou simples). Na versão mais atual, em sua extremidade distal (cabeça) existe um pequeno anel de náilon, com $2 \mathrm{~mm}$ de diâmetro, coberto de aço inoxidável, suturado na superfície interna do poliuretano, para tornar o patch visível à fluoroscopia. Na porção proximal (cauda), existe um pequeno fio metálico radiopaco que também auxilia a visualização do retalho à fluoroscopia (Figura 7). A ausência de esqueleto metálico e a forma do retalho the conferem grande flexibilidade e versatilidade, podendo ser aplicado a uma ampla variedade de defeitos, como CIA FO com bordas deficientes, CIA tipo seio venoso $(\mathrm{CIA} \mathrm{SV})^{4,5}$, comunicação interventricular perimembranosa (CIV PM), canais arteriais persistentes $(\mathrm{PCA})^{6}$ e, até mesmo, CIV de mau alinhamento ${ }^{7}$.

A essência do procedimento se baseia na possibilidade de oclusão do defeito pelo balão. O balão completamente inflado expande o retalho e o faz aderir às bordas do defeito e se manter estável. Depois de um tempo previamente estabelecido, de acordo com o procedimento realizado, o balão pode ser cuidadosamente retirado, deixando o patch fechando o orifício.

Quando não se obtém a completa oclusão com o balão, o procedimento se torna inviável. Essa característica confere segurança ao procedimento, possibilitando que novas opções de oclusão sejam tentadas para diferentes defeitos, além dos tradicionalmente fechados por via percutânea. Consideramos que essa é uma característica positiva do método.

A aderência do retalho às bordas do defeito se dá por meio da presença de fibrina e do aparecimento de células inflamatórias, o que ocorre, normalmente, em 24 a 48 horas.

O uso de cola biológica como fator facilitador e de aceleração da fixação do patch mostrou abreviar

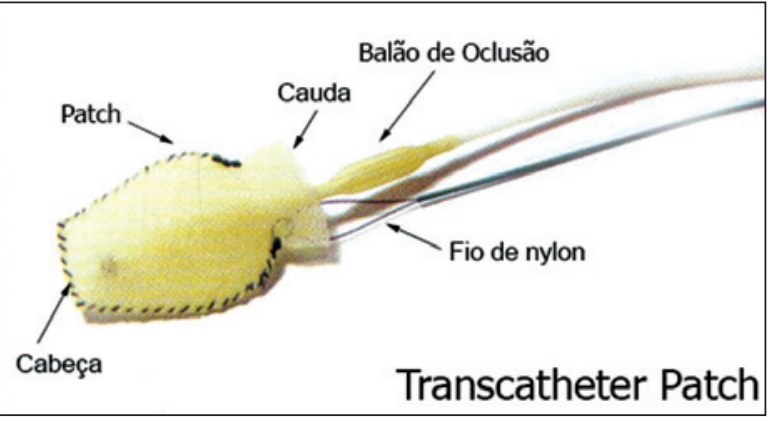

Figura 7 - Detalhes do patch. Visualiza-se a luva de poliuretano envolvendo o balão de oclusão e os demais detalhes do dispositivo. esse tempo. Nos defeitos com alto fluxo, como a CIV PM, observamos a fixação do dispositivo em apenas quatro horas. Para os defeitos com fluxos de mais baixa velocidade ou de arquitetura mais complexa, como o caso em questão, o tempo necessário para aderência do retalho, mesmo com o uso de cola, ainda aumenta consideravelmente. O caráter nãometálico e a porosidade do poliuretano empregado no retalho devem facilitar e abreviar sua endotelização.

Uma potencial desvantagem é que a cola biológica, utilizada em um dispositivo de uso intracardíaco introduzido percutaneamente, acrescenta ainda mais risco ao procedimento, embora não tenha sido relatada na literatura nenhuma complicação maior daí advinda.

A introdução do patch e a oclusão do defeito são, em geral, de fácil execução. A fase crítica do procedimento é a retirada do balão. Nesse procedimento, mesmo após a adequada obliteração do defeito, é impossível prever se o patch vai se manter no local, migrar ou mesmo sair aderido ao balão. $\mathrm{O}$ dado positivo é que a retirada de um dispositivo deslocado é extremamente fácil e dura apenas alguns segundos. O duplo fio de náilon mantém o retalho próximo à extremidade da bainha longa, impedindo sua embolização. O caráter flexível do tecido do retalho faz com que seja introduzido e retirado pela bainha longa com extrema facilidade e segurança.

O longo tempo de imobilização no leito, por vezes necessário para a liberação do balão, é outro dado negativo do procedimento.

\section{CONCLUSÕES}

Mesmo sendo um produto com registro aprovado pela Agência Nacional de Vigilância Sanitária (ANVISA) e, portanto, com venda liberada no País, ressaltamos que ainda é um procedimento experimental, e que necessita de mais estudos confirmando sua efetividade e segurança, em que pesem os bons resultados apresentados, até o momento, na literatura.

Embora relativamente simples e seguro, o implante do Transcatheter Patch $^{\circledR}$ apresenta certa complexidade e só deverá ser realizado em centros capacitados para tratamento percutâneo de defeitos congênitos, com profissionais adequadamente treinados e com experiência suficiente no uso de outras próteses.

O caráter, ainda artesanal, do produto com suas sucessivas e excessivas modificações, a falta de um protocolo para uniformização dos procedimentos, a ausência da reprodução dos bons resultados por outros operadores que não seu inventor e a falta de adequada documentação dos implantes por meio de imagens indiscutivelmente convincentes são outras causas de preocupação da comunidade científica, que, ainda hoje, mantém certo ceticismo e reluta em absorver essa nova técnica, apesar da grande aplicabilidade do método e de sua aparente eficácia e segurança. 

ou Apenas um Golpe de Sorte? Rev Bras Cardiol Invas. 2008;16(4):489-494.

\section{REFERÊNCIAS BIBLIOGRÁFICAS}

1. Ebels $\mathrm{T}$, Anderson RH. Atrioventricular septal defects. In: Anderson RH, Baker EJ, Macartney FJ, Rigby ML, Shinebourne EA, Tynan M, eds. Paediatric cardiology. $2^{\text {nd }}$ ed. London: Churchill Livingstone; 2002. p.939-81.

2. Harper RW, Mottram PM, McGaw DJ. Closure of secundum atrial septal defects with the Amplatzer septal occluder device: techniques and problems. Catheter Cardiovasc Interv. 2002;57(4):508-24.

3. Hein R, Büscheck F, Fischer E, Leetz M, Bayard MT, Ostermayer $S$, et al. Atrial and ventricular septal defects can safely be closed by percutaneous intervention. J Interv Cardiol. 2005; 18(6):515-22.
4. Sideris E, Toumanides S, Alekyan B, Varvarenko V, Stamatelopoulos S, Moulopoulos S. Transcatheter patch correction of atrial septal defects: experimental validation and early clinical experience. Circulation. 2000;102 Suppl II:II-588.

5. Sideris EB. Advances in transcatheter patch occlusion of heart defects. J Interv Cardiol. 2003;16(5):419-24.

6. Sideris EB, Macuil B, Varvarenko V, Alekyan B. Outpatient transcatheter patch occlusion of large patent ductus arteriosus and perimembranous ventricular septal defect. Catheter Cardiovasc Interv. 2004;62:140-7.

7. Sideris EB, Macuil B, Justiniano S, Rao PS. Total percutaneous correction of a tetralogy of Fallot variant with dominant pulmonary valve stenosis. Heart. 2005;91(3):345-7. 